QUANTUM DYNAMICS

BANACH CENTER PUBLICATIONS, VOLUME 120

INSTITUTE OF MATHEMATICS

POLISH ACADEMY OF SCIENCES

WARSZAWA 2020

\title{
PULLBACKS OF GRAPH C*-ALGEBRAS FROM ADMISSIBLE PUSHOUTS OF GRAPHS
}

\author{
PIOTR M. HAJAC \\ Instytut Matematyczny Polskiej Akademii Nauk \\ Śniadeckich 8, 00-656 Warszawa, Poland \\ and \\ Department of Mathematics, University of Colorado Boulder \\ 2300 Colorado Avenue, Boulder, CO 80309-0395, USA \\ ORCID: 0000-0001-9764-5697_E-mail: pmh@impan.pl \\ SARAH REZNIKOFF \\ Mathematics Department, Kansas State University \\ 138 Cardwell Hall, Manhattan, KS 66502, USA \\ ORCID: 0000-0001-8640-5890_E-mail: sarahrez@ksu.edu \\ MARIUSZ TOBOLSKI \\ Instytut Matematyczny Polskiej Akademii Nauk \\ Śniadeckich 8, 00-656 Warszawa, Poland \\ ORCID:0000-0001-7738-8515_E-mail: mtobolski@impan.pl
}

\begin{abstract}
We define an admissible decomposition of a graph $E$ into subgraphs $F_{1}$ and $F_{2}$, and consider the intersection graph $F_{1} \cap F_{2}$ as a subgraph of both $F_{1}$ and $F_{2}$. We prove that, if the decomposition of the graph $E$ into the subgraphs $F_{1}$ and $F_{2}$ is admissible, then the graph $\mathrm{C}^{*}$-algebra $C^{*}(E)$ of $E$ is the pullback $\mathrm{C}^{*}$-algebra of the canonical surjections from $C^{*}\left(F_{1}\right)$ and $C^{*}\left(F_{2}\right)$ onto $C^{*}\left(F_{1} \cap F_{2}\right)$.
\end{abstract}

2010 Mathematics Subject Classification: 46L55, 46L85.

Key words and phrases: pushout of graphs, pullback of algebras, gauge action, quantum spaces: spheres, balls, lens spaces, weighted projective spaces.

The paper is in final form and no version of it will be published elsewhere. 
1. Introduction and preliminaries. Pushouts of graphs have proven to be very useful in the theory of free groups 13 . We hope that our approach to pullbacks of graph algebras through pushouts of underlying graphs will also turn out to be beneficial.

A graph $\mathrm{C}^{*}$-algebra is the universal $\mathrm{C}^{*}$-algebra associated to a directed graph. If one considers a specific class of morphisms of directed graphs (e.g., see [1, Definition 1.6.2]), then the graph $\mathrm{C}^{*}$-algebra construction yields a covariant functor from the category of directed graphs to the category of $\mathrm{C}^{*}$-algebras. On the other hand, Hong and Szymański [8] showed that a pushout diagram in the category of directed graphs can lead to a pullback of $\mathrm{C}^{*}$-algebras. The purpose of this paper is to find conditions on the pushout diagram of graphs that give rise to the pullback diagram of the associated graph $\mathrm{C}^{*}$-algebras. This leads to a notion of an admissible decomposition of a directed graph, which we present in Section 2. The main result is contained in Section 3 and examples are in Section 4.

Our result is closely related to [9, Corollary 3.4], where it is proven, in an appropriate form, for $k$-graphs without sinks. Herein, we focus our attention on 1-graphs but possibly with sinks. Thus our results are complementary and lead to the following question: Is it possible to get rid of both of these assumptions ("only 1-graphs" and "no sinks") at the same time to prove a more general pushout-to-pullback theorem?

In this paper, by a graph $E$ we will always mean a directed graph, i.e. a quadruple $\left(E^{0}, E^{1}, s_{E}, r_{E}\right)$, where $E^{0}$ is the set of vertices, $E^{1}$ is the set of edges, $s_{E}: E^{1} \rightarrow E^{0}$ is the source map and $r_{E}: E^{1} \rightarrow E^{0}$ is the range map. (The sets $E^{0}$ and $E^{1}$ are usually assumed to be countable.) A graph $E$ is called row finite if each vertex emits only a finite number of edges. Next, $E$ is called finite if both $E^{0}$ and $E^{1}$ are finite. A vertex is called a sink in $E$ if $s_{E}^{-1}(v)=\varnothing$. By a finite path $\mu$ in $E$ we mean a finite sequence of composable edges $\mu:=$ $e_{1} e_{2} \ldots e_{k}$ or a vertex. The length $|\mu|$ of the finite path $\mu$ is the number $k$ of composable edges. We treat vertices as paths of length zero. The set of all finite paths for a graph $E$ is denoted by $\operatorname{Path}(E)$. One extends the source and the range maps to $\operatorname{Path}(E)$ in a natural way. We denote the extended source and range maps by $s_{P E}$ and $r_{P E}$, respectively.

Definition 1.1. The graph $C^{*}$-algebra $C^{*}(E)$ of a graph $E$ is the universal $C^{*}$-algebra generated by mutually orthogonal projections $P:=\left\{P_{v} \mid v \in E^{0}\right\}$ and partial isometries $S:=\left\{S_{e} \mid e \in E^{1}\right\}$ with mutually orthogonal ranges satisfying the Cuntz-Krieger relations [4, 6]:

$$
\begin{array}{cl}
S_{e}^{*} S_{e}=P_{r_{E}(e)} & \text { for all } e \in E^{1}, \\
\sum_{e \in s_{E}^{-1}(v)} S_{e} S_{e}^{*}=P_{v} & \text { for all } v \in E^{0} \text { that are not sinks, } \\
S_{e} S_{e}^{*} \leq P_{s_{E}(e)} & \text { for all } e \in E^{1} .
\end{array}
$$

The datum $\{S, P\}$ is called a Cuntz-Krieger E-family.

One can show that the above relations imply the standard path-algebraic relations:

$$
S_{f}^{*} S_{e}=0 \quad \text { for } \quad e \neq f, \quad P_{s_{E}(e)} S_{e}=S_{e}=S_{e} P_{r_{E}(e)} .
$$

Any graph $\mathrm{C}^{*}$-algebra $C^{*}(E)$ can be endowed with a natural circle action (called the gauge action)

$$
\alpha: U(1) \longrightarrow \operatorname{Aut}\left(C^{*}(E)\right)
$$


defined by its values on the generators:

$$
\alpha_{\lambda}\left(P_{v}\right)=P_{v}, \quad \alpha_{\lambda}\left(S_{e}\right)=\lambda S_{e}, \quad \text { where } \quad \lambda \in U(1), \quad v \in E^{0}, \quad e \in E^{1} .
$$

A subset $H$ of $E^{0}$ is called hereditary iff, for any $v \in H$ such that there is a path starting at $v$ and ending at $w \in E^{0}$, we have $w \in H$.

REMARK 1.2. One can equivalently define the property of being hereditary by replacing "path" with "edge".

Next, a subset $H$ of $E^{0}$ is called saturated iff there does not exist a vertex $v \notin H$ such that

$$
0<\left|s_{E}^{-1}(v)\right|<\infty \quad \text { and } \quad r_{E}\left(s_{E}^{-1}(v)\right) \subseteq H .
$$

Finally, a subset $H$ of $E^{0}$ is called unbroken iff there does not exist a vertex $v \notin H$ (called a breaking vertex in [1, Definition 2.4.4]) such that it emits infinitely many edges and

$$
0<\left|\left\{e \in s_{E}^{-1}(v) \mid r_{E}\left(s_{E}^{-1}(v)\right) \subseteq E^{0} \backslash H\right\}\right|<\infty .
$$

Unbroken saturated hereditary subsets play a fundamental role in the theory of gaugeinvariant ideals of graph $\mathrm{C}^{*}$-algebras. It follows from [2, p. 1163] that, for any hereditary subset $H$, the algebraic ideal generated by $\left\{P_{v} \mid v \in H\right\}$ is of the form

$$
I_{E}(H):=\operatorname{span}\left\{S_{x} S_{y}^{*} \mid x, y \in \operatorname{Path}(E), r_{P E}(x)=r_{P E}(y) \in H\right\} .
$$

Here, for any path $\mu=e_{1} \ldots e_{k}$, we adopt the notation $S_{\mu}:=S_{e_{1}} \ldots S_{e_{k}}$. Furthermore, if $\mu$ is a vertex, then $S_{\mu}:=P_{\mu}$.

By [2, Proposition 3.4], quotients by closed ideals generated by unbroken saturated hereditary subsets can also be realised as graph $\mathrm{C}^{*}$-algebras by constructing a quotient graph. Given a hereditary subset $H$ of $E^{0}$, the quotient graph $E / H$ is given by

$$
(E / H)^{0}:=E^{0} \backslash H \quad \text { and } \quad(E / H)^{1}:=E^{1} \backslash r_{E}^{-1}(H) .
$$

Note that the restriction-corestriction of the range map $r_{E}$ to $(E / H)^{1} \rightarrow(E / H)^{0}$ makes sense for any $H$, but the same restriction-corestriction of the source map $s_{E}$ exists because $H$ is hereditary. Moreover, if $H$ is also saturated and unbroken, we obtain the *-isomorphism

$$
C^{*}(E) / \overline{I_{E}(H)} \cong C^{*}(E / H)
$$

where $\overline{I_{E}(H)}$ is the norm closure of $I_{E}(H)$.

2. Admissible decompositions of graphs. Given two graphs $E=\left(E^{0}, E^{1}, s_{E}, r_{E}\right)$ and $G=\left(G^{0}, G^{1}, s_{G}, r_{G}\right)$, one can define a graph morphism $f: E \rightarrow G$ as a pair of mappings $f^{0}: E^{0} \rightarrow G^{0}$ and $f^{1}: E^{1} \rightarrow G^{1}$ satisfying

$$
s_{G} \circ f^{1}=f^{0} \circ s_{E} \quad \text { and } \quad r_{G} \circ f^{1}=f^{0} \circ r_{E} .
$$

We call the thus obtained category the category of directed graphs.

A subgraph of a graph $E=\left(E^{0}, E^{1}, s_{E}, r_{E}\right)$ is a graph $F=\left(F^{0}, F^{1}, s_{F}, r_{F}\right)$ such that

$$
F^{0} \subseteq E^{0}, \quad F^{1} \subseteq E^{1}, \quad \forall e \in F^{1}: s_{F}(e)=s_{E}(e) \text { and } r_{F}(e)=r_{E}(e) .
$$

Next, let $F_{1}$ and $F_{2}$ be two subgraphs of a graph $E$. We define their intersection and union as follows: 


$$
\begin{gathered}
F_{1} \cap F_{2}:=\left(F_{1}^{0} \cap F_{2}^{0}, F_{1}^{1} \cap F_{2}^{1}, s_{\cap}, r_{\cap}\right), \\
\forall e \in F_{1}^{1} \cap F_{2}^{1}: s_{\cap}(e):=s_{E}(e), r_{\cap}(e):=r_{E}(e), \\
F_{1} \cup F_{2}:=\left(F_{1}^{0} \cup F_{2}^{0}, F_{1}^{1} \cup F_{2}^{1}, s_{\cup}, r_{\cup}\right), \\
\forall e \in F_{1}^{1} \cup F_{2}^{1}: s_{\cup}(e):=s_{E}(e), r_{\cup}(e):=r_{E}(e) .
\end{gathered}
$$

Let us recall the pushout construction in the category of sets and directed graphs following [5]. Assume that $X, Y$ and $Z$ are sets and that $f: Z \rightarrow X$ and $g: Z \rightarrow Y$ are mappings. The pushout $P$ of $f$ and $g$ is defined by $P:=(X \sqcup Y) / \sim$, where $\sim$ is the finest equivalence relation such that $f(z) \sim g(z)$ for all $z \in Z$. If the mappings $f$ and $g$ are understood, we simply say that $P$ is a pushout of $X$ and $Y$ over $Z$. Additionally, we call the diagram

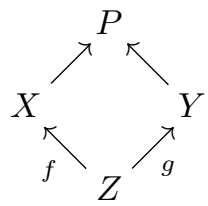

a pushout diagram of $X$ and $Y$ over $Z$. Here the upper arrows are compositions of the natural mappings from $X$ and $Y$ into their disjoint union followed by the quotient map $X \sqcup Y \rightarrow P$.

Directed graphs are defined in terms of sets, and therefore the pushout construction can be applied separately to the set of vertices and the set of edges. The only restriction is that all the mappings used need to preserve the source map and the range map. If a graph $E$ has two subgraphs $F_{1}$ and $F_{2}$ such that $E=F_{1} \cup F_{2}$, then the diagram

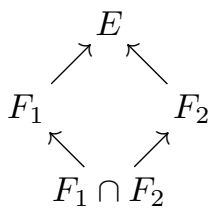

is automatically a pushout diagram of $F_{1}$ and $F_{2}$ over their intersection. Let us illustrate the concept of a pushout diagram of graphs with the following example:

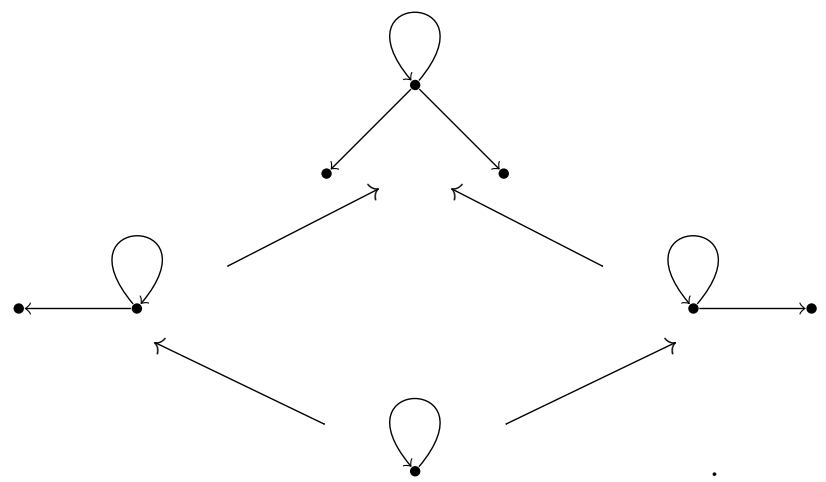


We are now ready to define an admissible decomposition of a graph:

Definition 2.1. An unordered pair $\left\{F_{1}, F_{2}\right\}$ of subgraphs of a graph $E$ is called an admissible decomposition of $E$ iff the following conditions are satisfied:

(1) $E=F_{1} \cup F_{2}$,

(2) if $v$ is a sink in $F_{1} \cap F_{2}$, then $v$ is a sink in $F_{i}, i=1,2$,

(3) $F_{1}^{1} \cap F_{2}^{1}=r_{F_{i}}^{-1}\left(F_{1}^{0} \cap F_{2}^{0}\right), i=1,2$,

(4) $F_{i}^{0} \backslash F_{j}^{0}$ is unbroken in $F_{i}^{0}$ and $E^{0}, i \neq j, i, j=1,2$.

Observe that Diagram 2.6 gives an example of an admissible decomposition of a graph. Note also that if $\left\{F_{1}, F_{2}\right\}$ is an admissible decomposition of $E$, then $E$ is automatically a pushout of $F_{1}$ and $F_{2}$ over their intersection by (1) in Definition 2.1. The goal of this paper is to use the other admissibility conditions to obtain a pullback diagram of the associated graph $\mathrm{C}^{*}$-algebras.

Definition 2.1 prompts the following two lemmas.

LEMMA 2.2. Let $\left\{F_{1}, F_{2}\right\}$ be an admissible decomposition of a graph $E$. Then we have $F_{1} \cap F_{2}=F_{i} /\left(F_{i}^{0} \backslash\left(F_{1}^{0} \cap F_{2}^{0}\right)\right)$ and $F_{i}=E /\left(E^{0} \backslash F_{i}^{0}\right)$, for $i=1,2$.

Proof. First, note that $F_{i}^{0} \backslash\left(F_{1}^{0} \cap F_{2}^{0}\right)$ is hereditary in $F_{i}$. Indeed, take $e \in F_{i}^{1}$. Then $s_{F_{i}}(e) \notin F_{1}^{0} \cap F_{2}^{0}$ implies $e \notin F_{1}^{1} \cap F_{2}^{1}$. Hence, by Definition 2.1 $(3), r_{F_{i}}(e) \notin F_{1}^{0} \cap F_{2}^{0}$. By Remark 1.2 we are done. Therefore, we can define $F_{i} /\left(F_{i}^{0} \backslash\left(F_{1}^{0} \cap F_{2}^{0}\right)\right)$, which coincides with $F_{1} \cap F_{2}$ due to Definition 2.1 (3).

Next, note that

$$
E^{0} \backslash F_{i}^{0}=\left(F_{i}^{0} \cup F_{j}^{0}\right) \backslash F_{i}^{0}=F_{j}^{0} \backslash F_{i}^{0}=F_{j}^{0} \backslash\left(F_{i}^{0} \cap F_{j}^{0}\right),
$$

where $j \neq i$ and $j=1,2$, so we already know that $E^{0} \backslash F_{i}^{0}$ is hereditary in $F_{j}$. To see that it is hereditary in $E$, we only need to exclude edges starting in $E^{0} \backslash F_{i}^{0}$ and ending in $E^{0} \backslash F_{j}^{0}$. They do not exist because $E^{1}=F_{i}^{1} \cup F_{j}^{1}$, so $E^{0} \backslash F_{i}^{0}$ is hereditary in $E$.

It remains to verify that $F_{i}^{1}=r_{E}^{-1}\left(F_{i}^{0}\right)$. To this end, taking advantage of the admissibility of $\left(F_{i} \cap F_{j}\right) \subseteq F_{i}$, we compute

$$
r_{E}^{-1}\left(F_{i}^{0}\right) \backslash F_{i}^{1}=r_{F_{j}}^{-1}\left(F_{i}^{0}\right) \backslash F_{i}^{1}=r_{F_{j}}^{-1}\left(F_{i}^{0} \cap F_{j}^{0}\right) \backslash F_{i}^{1}=\left(F_{i}^{1} \cap F_{j}^{1}\right) \backslash F_{i}^{1}=\varnothing .
$$

Therefore, as $F_{i}^{1} \subseteq r_{E}^{-1}\left(F_{i}^{0}\right)$, we conclude that $F_{i}^{1}=r_{E}^{-1}\left(F_{i}^{0}\right)$, as desired.

Lemma 2.3. Let $\left\{F_{1}, F_{2}\right\}$ be an admissible decomposition of a graph $E$. Then the subset $F_{i}^{0} \backslash\left(F_{1}^{0} \cap F_{2}^{0}\right)$ is saturated in $F_{i}^{0}$ and in $E^{0}$ for $i=1,2$.

Proof. If $F_{i}^{0} \backslash\left(F_{1}^{0} \cap F_{2}^{0}\right)$ were not saturated in $F_{i}^{0}$, then there would exist a vertex $v$ in

$$
F_{i}^{0} \backslash\left(F_{i}^{0} \backslash\left(F_{1}^{0} \cap F_{2}^{0}\right)\right)=F_{1}^{0} \cap F_{2}^{0}
$$

such that

$$
s_{F_{i}}^{-1}(v) \neq \varnothing \quad \text { and } \quad r_{F_{i}}\left(s_{F_{i}}^{-1}(v)\right) \subseteq F_{i}^{0} \backslash\left(F_{1}^{0} \cap F_{2}^{0}\right) .
$$

Thus we would have a vertex in $F_{1} \cap F_{2}$ that is a sink in $F_{1} \cap F_{2}$ but not in $F_{i}$, which contradicts Definition 2.1.2).

Much in the same way, suppose that $F_{i}^{0} \backslash\left(F_{1}^{0} \cap F_{2}^{0}\right)$ is not saturated in $E^{0}$. Then there exists a vertex

$$
w \in E^{0} \backslash\left(F_{i}^{0} \backslash\left(F_{1} \cap F_{2}^{0}\right)\right)=F_{j}^{0},
$$


where $j \neq i$ and $j=1,2$, such that

$$
s_{E}^{-1}(w) \neq \varnothing \quad \text { and } \quad r_{E}\left(s_{E}^{-1}(w)\right) \subseteq F_{i}^{0} \backslash\left(F_{1}^{0} \cap F_{2}^{0}\right) .
$$

Hence, there is $e \in s_{E}^{-1}(w)$ such that $r_{E}(e) \notin F_{j}^{0}$. As $E^{1}=F_{i}^{1} \cup F_{j}^{1}$, it follows that $e \in F_{i}^{1}$, so $w=s_{E}(e) \in F_{i}^{0}$. Consequently, $w$ is a sink in $F_{1} \cap F_{2}$ but not in $F_{i}$, which again contradicts Definition 2.1,2.

3. Pullbacks of graph $\mathbf{C}^{*}$-algebras. Let $\left\{F_{1}, F_{2}\right\}$ be an admissible decomposition of a graph $E$. Then, by Lemma 2.2 and Lemma 2.3 , we can take advantage of the formula (1.8) to define the canonical quotient maps:

$$
\begin{gathered}
\pi_{1}: C^{*}(E) \longrightarrow C^{*}(E) / \overline{I_{E}\left(F_{2}^{0} \backslash F_{1}^{0}\right)} \cong C^{*}\left(F_{1}\right), \\
\pi_{2}: C^{*}(E) \longrightarrow C^{*}(E) / \overline{I_{E}\left(F_{1}^{0} \backslash F_{2}^{0}\right)} \cong C^{*}\left(F_{2}\right), \\
\chi_{1}: C^{*}\left(F_{1}\right) \longrightarrow C^{*}\left(F_{1}\right) / \overline{I_{F_{1}}\left(F_{1}^{0} \backslash\left(F_{1}^{0} \cap F_{2}^{0}\right)\right)} \cong C^{*}\left(F_{1} \cap F_{2}\right), \\
\chi_{2}: C^{*}\left(F_{2}\right) \longrightarrow C^{*}\left(F_{2}\right) / \overline{I_{F_{2}}\left(F_{2}^{0} \backslash\left(F_{1}^{0} \cap F_{2}^{0}\right)\right)} \cong C^{*}\left(F_{1} \cap F_{2}\right) .
\end{gathered}
$$

Note that quotient maps are automatically $U(1)$-equivariant for the gauge action.

This brings us to the main theorem:

THEOREM 3.1. Let $\left\{F_{1}, F_{2}\right\}$ be an admissible decomposition of a graph E. Then there exist canonical quotient gauge-equivariant $*$-homomorphisms rendering the diagram

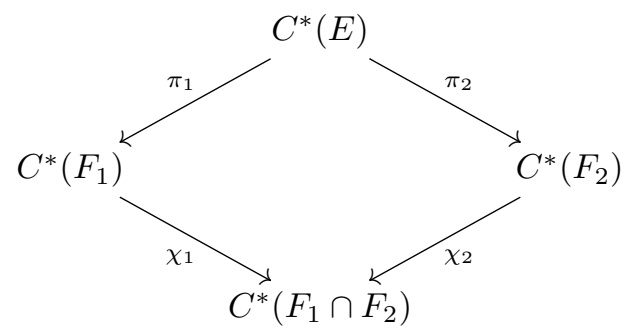

commutative. Moreover, this is a pullback diagram of $U(1)-C^{*}$-algebras.

Proof. Note first that all the canonical surjections in the diagram are well defined due to the admissibility conditions of the decomposition of the graph $E$ (see the discussion at the beginning of this section). The commutativity of the diagram is obvious as all maps are canonical surjections. Finally, using [10, Proposition 3.1] and the surjectivity of $\chi_{1}$ and $\chi_{2}$, to prove that $(3.2)$ is a pullback diagram, it suffices to show that $\operatorname{ker} \pi_{1} \cap \operatorname{ker} \pi_{2}=\{0\}$ and that $\operatorname{ker} \chi_{2} \subseteq \pi_{2}\left(\operatorname{ker} \pi_{1}\right)$.

Since ker $\pi_{1}$ and ker $\pi_{2}$ are closed ideals in a $C^{*}$-algebra, we know that

$$
\operatorname{ker} \pi_{1} \cap \operatorname{ker} \pi_{2}=\operatorname{ker} \pi_{1} \operatorname{ker} \pi_{2} .
$$

Next, as $F_{1}^{0} \backslash F_{2}^{0}$ and $F_{2}^{0} \backslash F_{1}^{0}$ are saturated hereditary subsets of $E^{0}$, it follows from (1.8) that

$$
\operatorname{ker} \pi_{1}=\overline{I_{E}\left(F_{2}^{0} \backslash F_{1}^{0}\right)} \quad \text { and } \quad \operatorname{ker} \pi_{2}=\overline{I_{E}\left(F_{1}^{0} \backslash F_{2}^{0}\right)} .
$$

Furthermore, using the characterization 1.6 of ideals generated by hereditary subsets, we know that an arbitrary element of $\operatorname{ker} \pi_{1} \operatorname{ker} \pi_{2}$ is in the closed linear span of elements 
of the form $S_{\alpha} S_{\beta}^{*} S_{\gamma} S_{\delta}^{*}$, where $\alpha, \beta \in \operatorname{Path}(E)$ with

$$
r_{P E}(\alpha)=r_{P E}(\beta) \in F_{2}^{0} \backslash F_{1}^{0},
$$

and $\gamma, \delta \in \operatorname{Path}(E)$ with

$$
r_{P E}(\gamma)=r_{P E}(\delta) \in F_{1}^{0} \backslash F_{2}^{0} .
$$

The conclusion ker $\pi_{1} \cap$ ker $\pi_{2}=\{0\}$ follows from the analysis of all possible paths satisfying the above conditions. Indeed, it follows from Definition 1.1 that $S_{\beta}^{*} S_{\gamma} \neq 0$ is possible only if $s_{P E}(\beta)=s_{P E}(\gamma)$. As $E^{1}=F_{1}^{1} \cup F_{2}^{1}, r_{P E}(\beta) \in F_{2}^{0} \backslash F_{1}^{0}$ and $r_{P E}(\gamma) \in F_{1}^{0} \backslash F_{2}^{0}$, if $\beta=e_{1} \ldots e_{m}$ and $\gamma=f_{1} \ldots f_{n}$, we infer that

$$
r_{E}\left(e_{m-1}\right)=s_{E}\left(e_{m}\right) \in F_{2}^{0} \quad \text { and } \quad r_{E}\left(f_{n-1}\right)=s_{E}\left(f_{n}\right) \in F_{1}^{0} .
$$

Hence $r_{E}\left(e_{m-1}\right) \in F_{1}^{0} \cap F_{2}^{0}$ or $r_{E}\left(e_{m-1}\right) \in F_{2}^{0} \backslash F_{1}^{0}$. Now, we continue by induction using Definition 2.1(3) for the intersection case of the alternative. This brings us to conclusion that $s_{P E}(\beta) \in F_{2}^{0}$. Much in the same way, we argue that $s_{P E}(\gamma) \in F_{1}^{0}$. It follows that $s_{P E}(\beta)=s_{P E}(\gamma) \in F_{1}^{0} \cap F_{2}^{0}$. Furthermore, as $r_{P E}(\beta) \in F_{2}^{0} \backslash F_{1}^{0}$ and $r_{P E}(\gamma) \in F_{1}^{0} \backslash F_{2}^{0}$, we conclude that $\beta \neq \gamma$, so there exists the smallest index $i$ such that $e_{i} \neq f_{i}$. Now, remembering the relation CK1 and (1.1), we compute

$$
\begin{aligned}
S_{\beta}^{*} S_{\gamma} & =S_{e_{i+1} \ldots e_{m}}^{*} S_{e_{i}}^{*} S_{e_{i-1}}^{*} \ldots S_{e_{1}}^{*} S_{e_{1}} \ldots S_{e_{i-1}} S_{f_{i}} S_{f_{i+1} \ldots f_{n}} \\
& =S_{e_{i+1} \ldots e_{m}}^{*} S_{e_{i}}^{*} S_{f_{i}} S_{f_{i+1} \ldots f_{n}}=0 .
\end{aligned}
$$

Finally, if $\beta$ or $\gamma$ is a path of length zero, i.e. a vertex, then it is straightforward to conclude that $S_{\beta}^{*} S_{\gamma}=0$.

Next, again taking advantage of (1.6) and (1.8), we obtain

$$
\operatorname{ker} \chi_{2}=\overline{I_{F_{2}}\left(F_{2}^{0} \backslash F_{1}^{0}\right)}=\overline{\operatorname{span}}\left\{S_{\alpha} S_{\beta}^{*} \mid \alpha, \beta \in \operatorname{Path}\left(F_{2}\right), r_{P F_{2}}(\alpha)=r_{P F_{2}}(\beta) \in F_{2}^{0} \backslash F_{1}^{0}\right\} \text {. }
$$

Any element of $I_{F_{2}}\left(F_{2}^{0} \backslash F_{1}^{0}\right)$ is an element of $I_{E}\left(F_{2}^{0} \backslash F_{1}^{0}\right)$, and $\pi_{2}\left(S_{\alpha}\right)=S_{\alpha}$ for all $\alpha \in \operatorname{Path}\left(F_{2}\right)$. Hence $I_{F_{2}}\left(F_{2}^{0} \backslash F_{1}^{0}\right) \subseteq \pi_{2}\left(I_{E}\left(F_{2}^{0} \backslash F_{1}^{0}\right)\right)$. Finally, from the continuity of $\pi_{2}$, we conclude that ker $\chi_{2} \subseteq \pi_{2}\left(\operatorname{ker} \pi_{1}\right)$.

REMARK 3.2. One can also prove Theorem 3.1 in the setting of Leavitt path algebras [1]. A proof of the Leavitt version of Theorem 3.1] is completely analogous due to [1, Corollary 2.5.11]. Furthermore, in the case of row-finite graphs, one should compare Theorem 3.1 with [12, Theorem 3.3].

4. Examples. We end the paper by providing motivating examples from noncommutative topology.

4.1. Even quantum spheres. Not only does the graph at the top of Diagram (2.6) representing the generic Podleś quantum sphere [11] admit a natural admissible decomposition, but also the finite graphs $L_{2 n}$ [7, Section 5.1] representing, respectively, the $\mathrm{C}^{*}$-algebras $C\left(S_{q}^{2 n}\right)$ of all even quantum spheres enjoy natural admissible decompositions $\left\{F_{2 n}^{1}, F_{2 n}^{2}\right\}$. Here $C^{*}\left(F_{2 n}^{1}\right)=C^{*}\left(F_{2 n}^{2}\right)$ coincides with the $\mathrm{C}^{*}$-algebra $C\left(B_{q}^{2 n}\right)$ of the Hong-Szymański quantum $2 n$-ball [8, Section 3.1], and $C^{*}\left(F_{2 n}^{1} \cap F_{2 n}^{2}\right)$ coincides [7, Section 4.1] with the $\mathrm{C}^{*}$-algebra $C\left(S_{q}^{2 n-1}\right)$ of the boundary Vaksman-Soibelman quantum odd sphere [14]. Thus we recover in terms of graphs the classical fact that an even sphere is a gluing of even balls over the boundary odd sphere. 
As Theorem 3.1 applies, we infer that the diagram

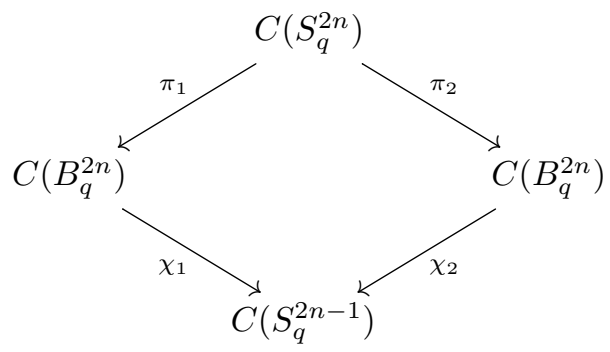

is a pullback diagram. This fact was already proved in [8, Proposition 5.1] by direct considerations of generators and relations.

The case $n=3$ is illustrated by the diagram:

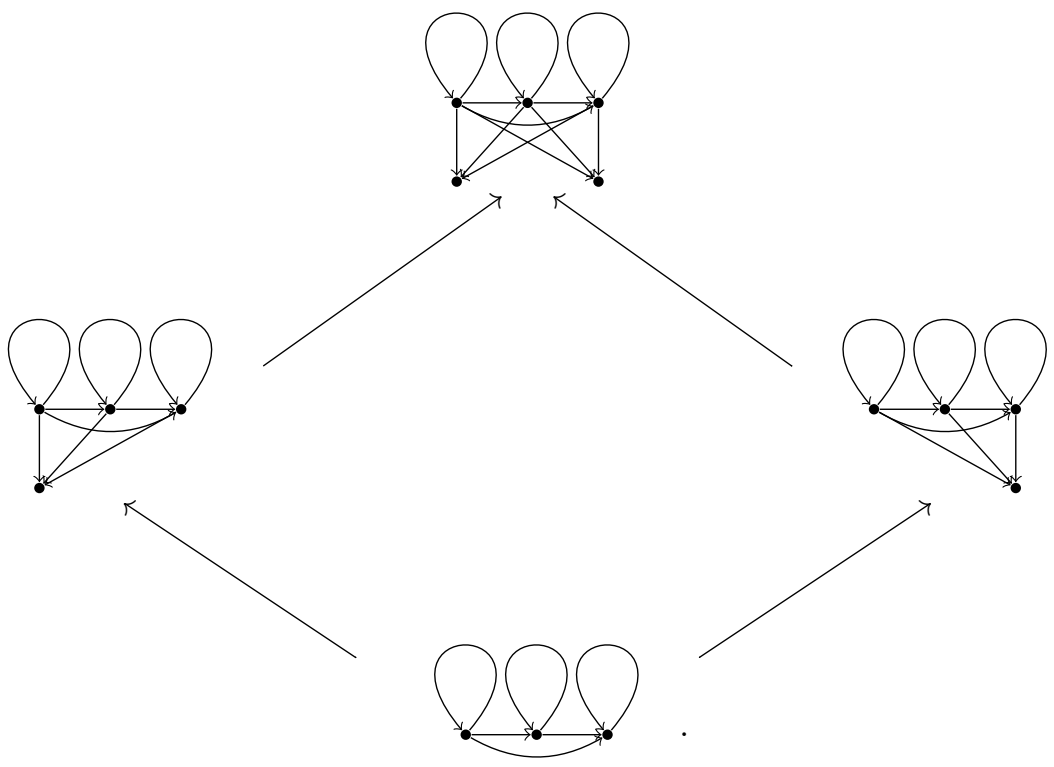

4.2. Quantum lens space $L_{q}^{3}(l ; 1, l)$. The $\mathrm{C}^{*}$-algebra $C\left(L_{q}^{3}(l ; 1, l)\right)$ of the quantum lens space $L_{q}^{3}(l ; 1, l)$ can be viewed as the graph $C^{*}$-algebra (e.g., see [3]) of the graph $L_{l}^{3}$ :

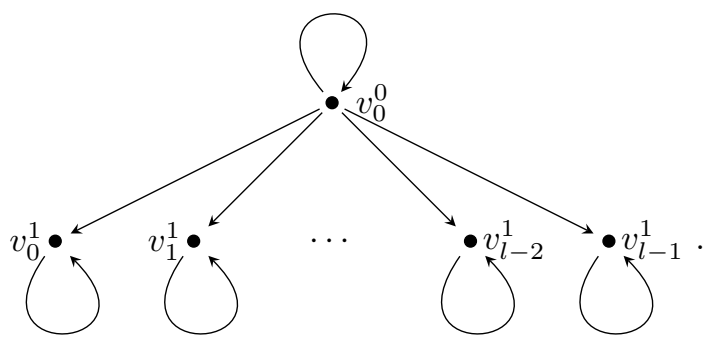


The graph $L_{l}^{3}$ enjoys an admissible decomposition $\left\{L_{k}^{3}, L_{l-k}^{3}\right\}$, where $k \in\{1, \ldots, l-1\}$, yielding, by Theorem 3.1 the pullback diagram:

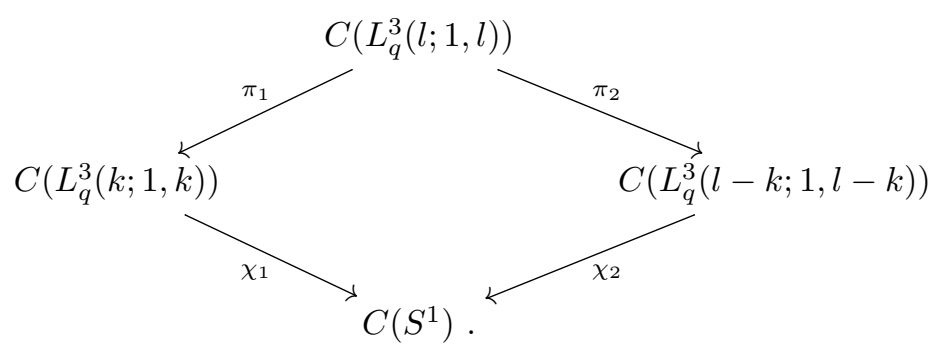

Recall that $C^{*}\left(L_{1}^{3}\right) \cong C\left(S_{q}^{3}\right)$, so, for $l=2$, we obtain the following pullback diagram:

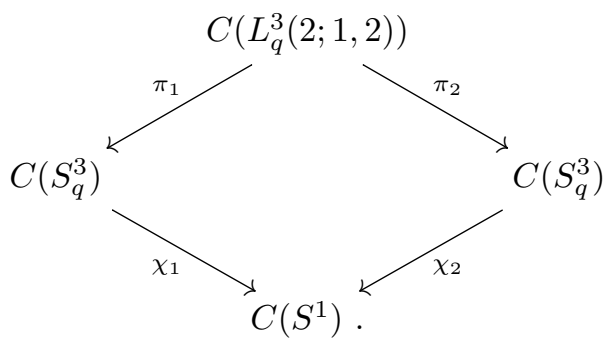

Since the above diagram is $U(1)$-equivariant, it induces a pullback diagram for $U(1)$-fixed-point subalgebras:

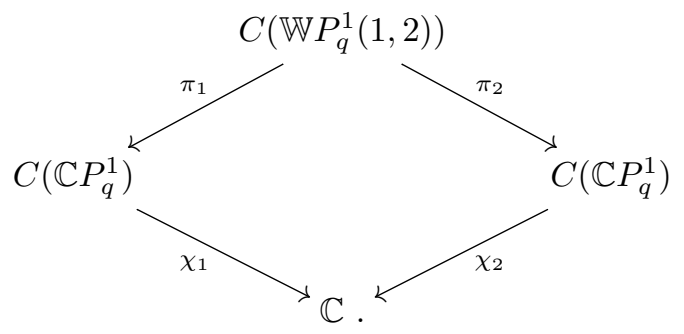

Here $C\left(\mathbb{C} P_{q}^{1}\right)$ and $C\left(\mathbb{W} P_{q}^{1}(1,2)\right)$ denote the quantum complex projective space (see [7, Section 2.3]) and the quantum weighted projective space (see [3, Section 3]), respectively. Interestingly, the $\mathrm{C}^{*}$-algebras in the above diagram can be viewed as graph $\mathrm{C}^{*}$-algebras, and an infinite graph representing $C\left(\mathbb{W} P_{q}^{1}(1,2)\right)$ is a pushout of infinite graphs representing $C\left(\mathbb{C} P_{q}^{1}\right)$ over the graph consisting of one vertex and no edges representing $\mathbb{C}$ (see Diagram 4.7 below). Better still, this example is also within the scope of Theorem 3.1

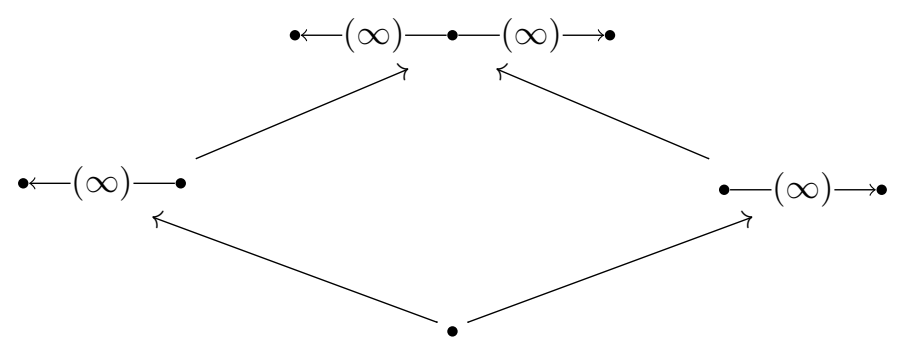

Here edges with $(\infty)$ denote countably infinitely many edges. 
Acknowledgements. The work on this project was partially supported by NCN-grant 2015/19/B/ST1/03098 (Piotr M. Hajac, Mariusz Tobolski) and by a Simons Foundation Collaboration Grant (Sarah Reznikoff). Piotr M. Hajac is very grateful to Kansas State University for its hospitality and financial support provided by this Simons Foundation Collaboration Grant. It is a pleasure to thank Carla Farsi for drawing our attention to using pushouts of graphs in the theory of free groups, Tatiana Gateva-Ivanova for a helpful discussion concerning admissible decompositions of graphs, and Aidan Sims for making us aware on how to prove our main result in a different context.

\section{References}

[1] G. Abrams, P. Ara, M. Siles Molina, Leavitt Path Algebras, Lecture Notes in Math. 2191, Springer, London, 2017.

[2] T. Bates, J. H. Hong, I. Raeburn, W. Szymański, The ideal structure of the $C^{*}$-algebras of infinite graphs, Illinois J. Math. 46 (2002), 1159-1176.

[3] T. Brzeziński, W. Szymański, The $C^{*}$-algebras of quantum lens and weighted projective spaces, J. Noncommut. Geom. 12 (2018), 195-215.

[4] J. Cuntz, W. Krieger, A class of $C^{*}$-algebras and topological Markov chains, Invent. Math. 56 (1980), 251-268.

[5] H. Ehrig, H.-J. Kreowski, Pushout-properties: an analysis of gluing constructions for graphs, Math. Nachr. 91 (1979), 135-149.

[6] N. J. Fowler, M. Laca, I. Raeburn, The $C^{*}$-algebras of infinite graphs, Proc. Amer. Math. Soc. 128 (2000), 2319-2327.

[7] J. H. Hong, W. Szymański, Quantum spheres and projective spaces as graph algebras, Comm. Math. Phys. 232 (2002), 157-188.

[8] J. H. Hong, W. Szymański, Noncommutative balls and mirror quantum spheres, J. Lond. Math. Soc. (2) 77 (2008), 607-626.

[9] A. Kumjian, D. Pask, A. Sims, M. F. Whittaker, Topological spaces associated to higher-rank graphs, J. Combin. Theory Ser. A 143 (2016), 19-41.

[10] G. K. Pedersen, Pullback and pushout constructions in $C^{*}$-algebra theory, J. Funct. Anal. 167 (1999), 243-344.

[11] P. Podleś, Quantum Spheres, Lett. Math. Phys. 14 (1987), 193-202.

[12] D. Robertson, W. Szymański, $C^{*}$-algebras associated to $C^{*}$-correspondences and applications to mirror quantum spheres, Illinois J. Math. 55 (2011), 845-870.

[13] J. R. Stallings, Topology of finite graphs, Invent. Math. 71 (1983), 551-565.

[14] L. L. Vaksman, Ya. S. Soủbel'man, Algebra of functions on the quantum group $\mathrm{SU}(\mathrm{n}+1)$ and odd-dimensional quantum spheres, Algebra i Analiz 2 (1990), no. 5, 101-120; English transl.: Leningrad Math. J. 2 (1991), 1023-1042. 